\title{
Serum Biomarker Identification by Mass Spectrometry in Acute Aortic Dissection
}

\author{
Yong Rena, Q Qizhu Tang ${ }^{a} \quad$ Wenwei Liub Yongqian Tang ${ }^{b} \quad$ Rui Zhub Bin Lib \\ aDepartment of Cardiology, Renmin Hospital of Wuhan University, Wuhan, bepartment of Cardiology, \\ Xiangyang Central Hospital, Affiliated Hospital of Hubei University of Arts and Science, Xiangyang, \\ P.R. China
}

\section{Key Words}

Aortic dissection • Metabolomics • Peripheral blood markers • HPLCMS

\begin{abstract}
Background/Aims: Aortic dissection (AD) is also known as intramural hematoma. This study aimed to screen peripheral blood biomarkers of small molecule metabolites for $A D$ using high-performance liquid chromatography-mass spectrometry (HPLC-MS). Methods: Sera from 25 healthy subjects, 25 patients with well-established AD, and 25 patients with well-established hypertension were investigated by HPLC-MS to detect metabolites, screen differentially expressed metabolites, and analyze metabolic pathways. Results: Twenty-six and four metabolites were significantly up- and down-regulated in the hypertensive patients compared with the healthy subjects; 165 metabolites were significantly up-regulated and 109 significantly down-regulated in the $A D$ patients compared with the hypertensive patients. Of these metabolites, 35 were up-regulated and 105 down-regulated only in AD patients. The metabolites that were differentially expressed in AD are mainly involved in tryptophan, histidine, glycerophospholipid, ether lipid, and choline metabolic pathways. As AD alters the peripheral blood metabolome, analysis of peripheral blood metabolites can be used in auxiliary diagnosis of AD. Conclusion: Eight metabolites are potential biomarkers for $A D, 3$ of which were differentially expressed and can be used for auxiliary diagnosis of $A D$ and evaluation of treatment effectiveness.

\section{Introduction}

Acute myocardial infarction, pulmonary embolism, and aortic dissection (AD) are not only common causes of clinical chest pain but also major diseases leading to death in those with acute chest pain [1-3]. AD is caused by tearing of the local endothelium, which is gradually stripped due to the impact of blood stream flow. This lesion expands, and two lumens (true and false) form within the aorta, resulting in manifestations that include tearlike pain $[4,5]$. The aorta, the trunk blood vessel of the body, directly bears the pressure 


\section{Cellular Physiology Cell Physiol Biochem 2017;44:2147-2157

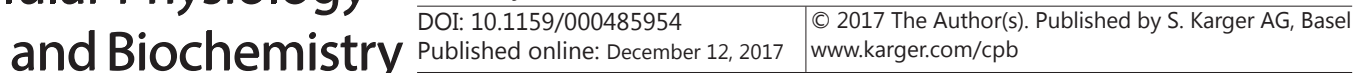 \\ Ren et al.: Serum Biomarkers for Aortic Dissection}

generated by the heart beat. The blood flow within the aorta is so strong that the chance of rupture is great in instances in which an endothelial-tearing lesion is not appropriately and timely treated, and the mortality rate is high [6]. The incidence of AD in the United States is 25-30/1 million [7]. In addition, the overall in-hospital mortality rate in China is reportedly $17.7 \%$ [8], and the number of patients with $\mathrm{AD}$ found in clinical practice in recent years is showing an obvious increasing trend.

At present, the main auxiliary diagnostic approaches for $\mathrm{AD}$ are computed tomographic angiography (CTA), magnetic resonance imaging (MRI), and digital subtraction angiography (DSA) [9-10]. However, these detection methods are employed only after disease onset, and radiation and contrast agents are harmful to the body. Although there are abundant peripheral blood biomarkers, the specificity and interpretation of these biomarkers in the setting of acute $\mathrm{AD}$ remains clinically challenging. In general, the pathological processes of $\mathrm{AD}$ are thought to include the initial inflammatory response, degradation and destruction of vascular wall components and their release into the peripheral blood, and activation of the coagulation mechanism [11]. Therefore, recent identification of early peripheral blood biomarkers for $\mathrm{AD}$ has focused on markers for vascular endothelial injury and repair and inflammatory reactions in the early stage of the disease $[12,13]$. The currently reported peripheral blood biomarkers for $\mathrm{AD}$ are all protein macromolecules, such as smooth muscle myosin heavy chain (smMHC), creatine kinase-BB (CK-BB), D-dimer, matrix metalloproteinases (MMPs), and elastin [14-16]. The discovery of smMHC was a landmark for early AD diagnosis: according to Suzuki et al., blood smMHC levels were dramatically elevated within 3 hours after the onset of acute AD, with a specificity up to $90.0 \%$ [14]. Additional protein molecules closely related to the development of $\mathrm{AD}$ have since been found. For instance, the level of lipoprotein (LP) is significantly higher in patients with AD than in patients with myocardial infarction or normal controls, possibly because LP facilitates inflammatory reactions in the arterial wall, thereby affecting its structure [15].

Although peripheral blood detection is a rapid and less-invasive technique, effective peripheral blood markers for AD are lacking, especially small molecule metabolite markers [17]. The existing peripheral blood biomarkers for AD include macromolecular proteins, yet only smMHC has gained public recognition to date. Moreover, detection of protein as an early diagnostic marker is expensive and not conducive to generalization. With the recent cross-development of biological, chemical, and software designs and the accumulation of databases, an increasing number of new diagnostic methods are being introduced for clinical diagnosis, especially peripheral blood diagnosis. Among these methods, high-performance liquid chromatography-mass spectrometry (HPLC-MS) has gradually been recognized as a screening method for serum biomarkers $[18,19]$.

Given the current status of research into AD-induced changes in whole-body metabolism, this study evaluated a simple and rapid testing approach. High-throughput screening of metabolites in the peripheral blood of patients with AD was performed by HPLC-MS to identify peripheral blood metabolite markers associated with $\mathrm{AD}$ and to explore possible metabolic pathways, analyze related metabolic pathways, and explain the possible pathogenesis of AD.

\section{Materials and Methods}

\section{Participants}

A total of $25 \mathrm{AD}$ patients who received treatment between May 23 and October 15, 2016, at Renmin Hospital of Wuhan University were randomly recruited. Inclusion criteria for the AD patients were as follows: 1) a confirmed diagnosis of AD via imaging data (CTA, DSA, or MRI) and 2) a time interval between the initial onset and sampling of less than or equal to 14 days. If a patient met both of the above criteria, he/she was enrolled in the AD group. Patients with the following conditions were excluded from the AD group: 1) various aortic traumata not caused by injury, such as aortic transection and aortic pseudoaneurysm; 2) AD patients with a time interval of more than 14 days from initial onset to sampling; and 3) patients with acute or chronic infectious diseases, autoimmune diseases or malignancy, a long history of immunosuppressive drug 


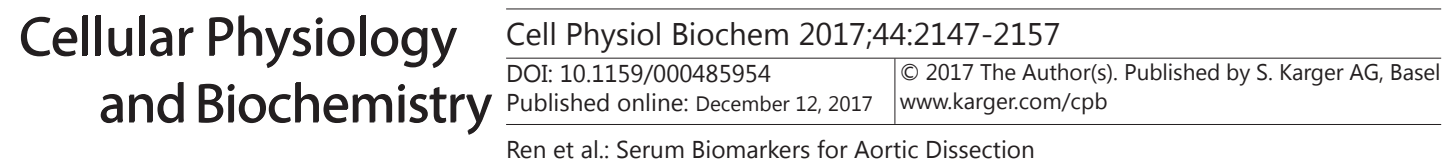

use, a history of venous thrombosis or myocardial infarction, and the presence of severe liver and kidney dysfunction or serious electrolyte disorders. Among the AD patients, only two were free from hypertension or inherited hypertension (HP). Clinical data for the AD group are summarized in Table 1.

HP patients (contractive pressure $\geq 140 \mathrm{mmHg}$ and/or diastolic pressure $\geq 90 \mathrm{mmHg}$ before hypertension treatment) were randomly selected from May 30 to November 23, 2016, including those with a confirmed diagnosis of hypertension in our hospital or hypertensive patients without other cardiovascular diseases according to a physical examination. Clinical data for the HP group are summarized in Table 2.

A normal control (NC) group was selected from May 30 to September 5, 2016, comprising normal adults without cardiovascular diseases or family genetic diseases. Their blood fat, blood glucose, blood pressure, and hepatonephric function indices were all within normal ranges.

This study was approved by the Ethics Committee of Renmin Hospital, and informed consent was obtained from each participant.

\section{Sample collection}

All whole-blood samples were collected at the hospital on the morning after confirmed diagnosis. Intravenous blood collection tubes were used to collect $1.5 \mathrm{~mL}$ of whole blood from each participant; the immediate clinical laboratory test required approximately $0.5 \mathrm{~mL}$, and the remaining $1.0 \mathrm{~mL}$ was used for serum separation. Each whole blood sample was incubated at $37^{\circ} \mathrm{C}$ for $1-2 \mathrm{~h}$ and centrifuged at $2000 \mathrm{r} / \mathrm{min}$ for $5 \mathrm{~min}$; the supernatant, considered the serum, was immediately frozen at $-80{ }^{\circ} \mathrm{C}$.
Table 1. The clinical information of AD patients. * No history of hypertension

\begin{tabular}{|c|c|c|c|c|c|c|c|c|c|c|}
\hline $\begin{array}{l}\text { Patient } \\
\text { No. }\end{array}$ & Gender & Age & TG & TC & HDL & LDL & $\begin{array}{c}\text { Pressure } \\
\text { value }\end{array}$ & $\begin{array}{l}\text { Blood } \\
\text { glucose }\end{array}$ & Urea & Creatinine \\
\hline 1 & Female & 58 & 0.99 & 4.75 & 1.51 & 2.73 & $145 / 90$ & 6.04 & 7.6 & 77.9 \\
\hline 2 & Female & 61 & 1.5 & 5.1 & 1.21 & 3.16 & $176 / 85$ & 5.8 & 7.2 & $70.8^{*}$ \\
\hline 3 & Male & 58 & 0.68 & 3.85 & 0.75 & 2.7 & $140 / 90$ & 6.67 & 5.7 & 69.4 \\
\hline 4 & Female & 66 & 0.77 & 4.35 & 0.6 & 3.18 & $150 / 80$ & 6.28 & 4.5 & 56 \\
\hline 5 & Male & 43 & 0.78 & 3.77 & 1.32 & 1.95 & $160 / 76$ & 5.47 & 8.2 & 124.9 \\
\hline 6 & Male & 54 & 1.13 & 2.58 & 0.57 & 1.45 & $100 / 60$ & 5.44 & 7.8 & 98.8 \\
\hline 7 & Male & 50 & 1.05 & 6.61 & 1.21 & 4.92 & $230 / 118$ & 5.85 & 6.57 & 69.5 \\
\hline 8 & Female & 62 & 1.14 & 4.73 & 1.07 & 3.06 & $202 / 112$ & 5.52 & 6.4 & 60.4 \\
\hline 9 & Male & 53 & 1.2 & 3.36 & 0.79 & 2.07 & $188 / 60$ & 7.01 & 8.1 & 94.1 \\
\hline 10 & Male & 54 & 1.21 & 3.76 & 0.89 & 2.3 & $180 / 86$ & & 4.6 & 118.7 \\
\hline 11 & Male & 52 & 2.54 & 5.12 & 0.71 & 3.38 & $112 / 66$ & 5.22 & 7.4 & 87.9 \\
\hline 12 & Male & 44 & 1.41 & 2.69 & 0.72 & 1.31 & $170 / 72$ & 8.72 & 8.8 & 108.9 \\
\hline 13 & Male & 61 & 1.46 & 3.85 & 1.08 & 2.28 & $118 / 80$ & 6.04 & 6.1 & 121.9 \\
\hline 14 & Male & 48 & 1.77 & 5.47 & 0.93 & 3.89 & $116 / 70$ & 5.38 & 4.6 & 61.1 \\
\hline 15 & Male & 46 & 0.58 & 3.92 & 1.09 & 2.49 & $154 / 90$ & 5.87 & 8 & 154.6 \\
\hline 16 & Female & 36 & 1.23 & 3.6 & 0.9 & 2.07 & $114 / 56$ & 5.26 & 24.9 & 920.1 \\
\hline 17 & Male & 40 & 0.7 & 4.46 & 0.96 & 3.11 & $164 / 88$ & 6.54 & 6.7 & 84 \\
\hline 18 & Male & 46 & 2.79 & 3.87 & 0.64 & 2.21 & $140 / 90$ & 4.99 & 6.3 & 79.8 \\
\hline 19 & Female & 60 & 0.65 & 3.67 & 1.06 & 2.2 & $160 / 90$ & 5.28 & 4.4 & 54.3 \\
\hline 20 & Male & 36 & 2 & 2.58 & 0.86 & 1.07 & $176 / 110$ & 5.14 & 6.6 & 78.7 \\
\hline 21 & Male & 42 & 0.72 & 2.9 & 0.96 & 1.57 & $170 / 106$ & 5.88 & 7.9 & 87.2 \\
\hline 22 & Male & 33 & 1.4 & 4.62 & 1.1 & 3.09 & $166 / 100$ & 4.94 & 7.3 & 60.8 \\
\hline 23 & Male & 49 & 1 & 4.66 & 1.67 & 2.65 & $138 / 74$ & 5.1 & 5.5 & $72.1^{*}$ \\
\hline 24 & Male & 61 & 1.51 & 4.53 & 1.04 & 3.18 & $116 / 72$ & 8.34 & 4.3 & 78.1 \\
\hline 25 & Male & 52 & 0.65 & 3.61 & 1.77 & 2.72 & $149 / 109$ & 5.13 & 6.7 & 88.9 \\
\hline 26 & Male & 54 & 1.2 & 4.36 & 1.22 & 1.97 & 198/90 & 6.01 & 8.6 & 92.9 \\
\hline 27 & Male & 63 & 1.41 & 2.99 & 0.79 & 2.8 & $186 / 83$ & 5.32 & 5.4 & 100.7 \\
\hline 28 & Male & 36 & 2.11 & 3.28 & 0.69 & 3.15 & $179 / 76$ & 5.17 & 7.5 & 84.3 \\
\hline 29 & Female & 43 & 2.09 & 4.92 & 0.83 & 1.99 & $150 / 72$ & 6.02 & 7.8 & 88.9 \\
\hline 30 & Female & 46 & 0.96 & 3.18 & 1.28 & 2.11 & $128 / 85$ & 7.33 & 5.9 & 107.3 \\
\hline
\end{tabular}

Table 2. The clinical information of HP patients

\begin{tabular}{|c|c|c|c|c|c|c|c|c|}
\hline $\begin{array}{l}\text { Patient } \\
\text { No. }\end{array}$ & Gender & Age & TG & TC & $\begin{array}{l}\text { Pressure } \\
\text { value }\end{array}$ & HDL & LDL & $\begin{array}{l}\text { Blood } \\
\text { glucose }\end{array}$ \\
\hline 1 & Female & 63 & 0.68 & 3.88 & $160 / 100$ & 1.26 & 2.12 & Normal \\
\hline 2 & Female & 58 & 1.72 & 5.86 & $150 / 100$ & 1.41 & 3.49 & Normal \\
\hline 3 & Female & 66 & 0.87 & 4.7 & $160 / 100$ & 1.02 & 3.17 & Normal \\
\hline 4 & Female & 68 & 1.09 & 6.5 & $140 / 100$ & 1.23 & 4.5 & 6.17 \\
\hline 5 & Female & 65 & 1.54 & 4.44 & $150 / 90$ & 1.24 & 2.26 & 7.37 \\
\hline 6 & Female & 40 & 1.53 & 6.87 & $150 / 90$ & 1.7 & 4.06 & 8.17 \\
\hline 7 & Female & 69 & 1.66 & 6.13 & $155 / 80$ & 1.46 & 3.68 & Normal \\
\hline 8 & Female & 69 & 1.91 & 6.39 & $160 / 90$ & 1.2 & 4.19 & 7.46 \\
\hline 9 & Female & 60 & 1.58 & 3.3 & $150 / 90$ & 1.19 & 1.33 & Normal \\
\hline 10 & Male & 67 & 2.99 & 5.74 & $180 / 86$ & 1.14 & 3.53 & Normal \\
\hline 11 & Male & 61 & 3.51 & 4.3 & $140 / 100$ & 0.78 & 2.43 & Normal \\
\hline 12 & Male & 52 & 1.99 & 5.09 & $140 / 100$ & 0.29 & 3.11 & Normal \\
\hline 13 & Male & 65 & 1.03 & 4.23 & $140 / 100$ & 1 & 2.82 & Normal \\
\hline 14 & Male & 57 & 3.51 & 4.47 & $130 / 94$ & 0.82 & 2.39 & Normal \\
\hline 15 & Female & 53 & 1.04 & 6.96 & $130 / 100$ & 1.58 & 4.55 & 7.25 \\
\hline 16 & Male & 53 & 1.26 & 5.6 & $138 / 96$ & 1.39 & 3.66 & Normal \\
\hline 17 & Female & 48 & 1.77 & 5.08 & $150 / 90$ & 1.16 & 2.91 & Normal \\
\hline 18 & Male & 57 & 10.67 & 6.3 & $140 / 90$ & 0.73 & 4.63 & 14.21 \\
\hline 19 & Female & 54 & 0.65 & 5.11 & $140 / 90$ & 1.69 & 2.94 & Normal \\
\hline 20 & Male & 62 & 0.93 & 3.74 & $150 / 100$ & 1.15 & 2 & Normal \\
\hline 21 & Male & 46 & 1.06 & 3.81 & $130 / 100$ & 1.17 & 2.08 & Normal \\
\hline 22 & Female & 54 & 0.86 & 4.57 & $130 / 94$ & 1.16 & 2.99 & Normal \\
\hline 23 & Male & 41 & 1.77 & 4.57 & $140 / 100$ & 1.16 & 2.81 & Normal \\
\hline 24 & Male & 45 & 1.85 & 5.83 & $150 / 120$ & 1.28 & 3.86 & Normal \\
\hline 25 & Male & 61 & 0.86 & 4.66 & $176 / 100$ & 1.27 & 2.91 & 11.36 \\
\hline 26 & Male & 52 & 1.7 & 4.45 & $130 / 100$ & 1.07 & 2.93 & Normal \\
\hline 27 & Male & 50 & 3.25 & 6.24 & $130 / 90$ & 1.11 & 3.68 & Normal \\
\hline 28 & Male & 41 & 2 & 4.35 & $120 / 100$ & 1.2 & 2.47 & Normal \\
\hline 29 & Male & 44 & 1.13 & 3.38 & $130 / 90$ & 1.34 & 1.56 & Normal \\
\hline 30 & Male & 53 & 0.91 & 5.3 & $140 / 90$ & 1.38 & 3.45 & Normal \\
\hline
\end{tabular}




\section{Cellular Physiology Cell Physiol Biochem 2017;44:2147-2157 \begin{tabular}{l|l|l} 
and Biochemistry & DOI: 10.1159/000485954 & $\begin{array}{l}\text { C } 2017 \text { The Author(s). Published by S. Karger AG, Basel } \\
\text { www.karger.com/cpb }\end{array}$
\end{tabular} \\ Ren et al.: Serum Biomarkers for Aortic Dissection}

\section{Sample pretreatment}

The solvents used as mobile phases in assays, including acetonitrile, methanol, isopropanol, and formic acid, were purchased from CNW Corporation, Germany, and unless otherwise indicated, were all of chromatographic grade. The water used in the experiments was Millipore ultrapure water. Serum samples were processed as follows. A total of $100 \mu \mathrm{L}$ serum was added to $0.3 \mathrm{~mL}$ methanol; the sample was shaken for $30 \mathrm{~s}$ and allowed to stand for $2 \mathrm{~min}$. The sample was subjected to ultrasound for 30 min and centrifugation at $12,000 \mathrm{rpm}$ at $4{ }^{\circ} \mathrm{C}$ for $10 \mathrm{~min}$, and $200-\mu \mathrm{L}$ aliquots were collected into sample vials and stored at $4{ }^{\circ} \mathrm{C}$ for measurement.
Table 3. Gradients of the HPLC-MS mobile phase

\begin{tabular}{llll}
\hline $\begin{array}{l}\text { Time } \\
(\mathrm{min})\end{array}$ & $\begin{array}{l}\text { Flow rate } \\
(\mathrm{mL} / \mathrm{min})\end{array}$ & $\begin{array}{l}\mathrm{A} \\
(\%)\end{array}$ & $\begin{array}{l}\mathrm{B} \\
(\%)\end{array}$ \\
\hline 0 & 0.3 & 95 & 5 \\
2 & 0.3 & 95 & 5 \\
12 & 0.3 & 5 & 95 \\
15 & 0.3 & 5 & 95 \\
17 & 0.3 & 95 & 5 \\
20 & 0.3 & 95 & 5 \\
\hline
\end{tabular}

\section{Mass spectrometry analysis parameters and conditions}

The chromatographic conditions were as follows. An Agilent QTOF-6545 system with a microfluidic high-performance liquid phase was used with Eclipse-C18 columns $(3 \times 100 \mathrm{~mm}, 1.8 \mu \mathrm{m})$. The column temperature was $40{ }^{\circ} \mathrm{C}$. Mobile phase A was water (V/V) (containing $0.1 \%$ formic acid), and mobile phase $\mathrm{B}$ was acetonitrile (containing $0.1 \%$ formic acid). The flow rate was $0.3 \mathrm{~mL} / \mathrm{min}$, and the injection volume was $4 \mu \mathrm{L}$; the mobile-phase gradients are shown in Table 3. The mass spectrometry conditions included an Agilent Jet Stream (AJS) source, the scanning mode of the electrospray ionization (ESI ${ }^{+}$) mode, capillary voltages of $1.4 \mathrm{kV}$ and $1.3 \mathrm{kV}$, cone voltages of $40 \mathrm{~V}$ and $23 \mathrm{~V}$, an ion source temperature of $20^{\circ} \mathrm{C}$, a desolvation gas temperature of $350{ }^{\circ} \mathrm{C}$, a cone gas flow of $10 \mathrm{~L} / \mathrm{h}$, a desolvation gas flow of $600 \mathrm{~L} / \mathrm{h}$, a collision energy of 10-40 V, and an ion energy of $1 \mathrm{~V}$. A chart was collected every $0.2 \mathrm{~s}$. Rutin solution was used as the fixedquantity solution for accurate mass determination. The mass scan range was 50-1500 m/z. The HPLC-MS mobile phase gradients are shown in Table 3.

\section{Instrument stability test}

A total of $2 \mu \mathrm{L}$ of each sample was mixed well and centrifuged, and $4 \mu \mathrm{L}$ of the supernatant was successively injected 6 times. Under the exact same test conditions, the stability of the instrument was examined according to the overlap of the original chromatograms. The stability of the database was evaluated, and analysis was performed according to the different types of metabolites obtained from the 6 injections.

\section{Statistical analysis}

Differences were analyzed with SPSS 20 software, and $P<0.05$ was considered significant. QTOF6545 software and MPP mass s p e c t r o m e t r y workstation software (Agilent) were used for composition extraction from each sample and data preprocessing, including baseline filtering, peak identification and integration, retention time correction, peak alignment, and mass spectrometry

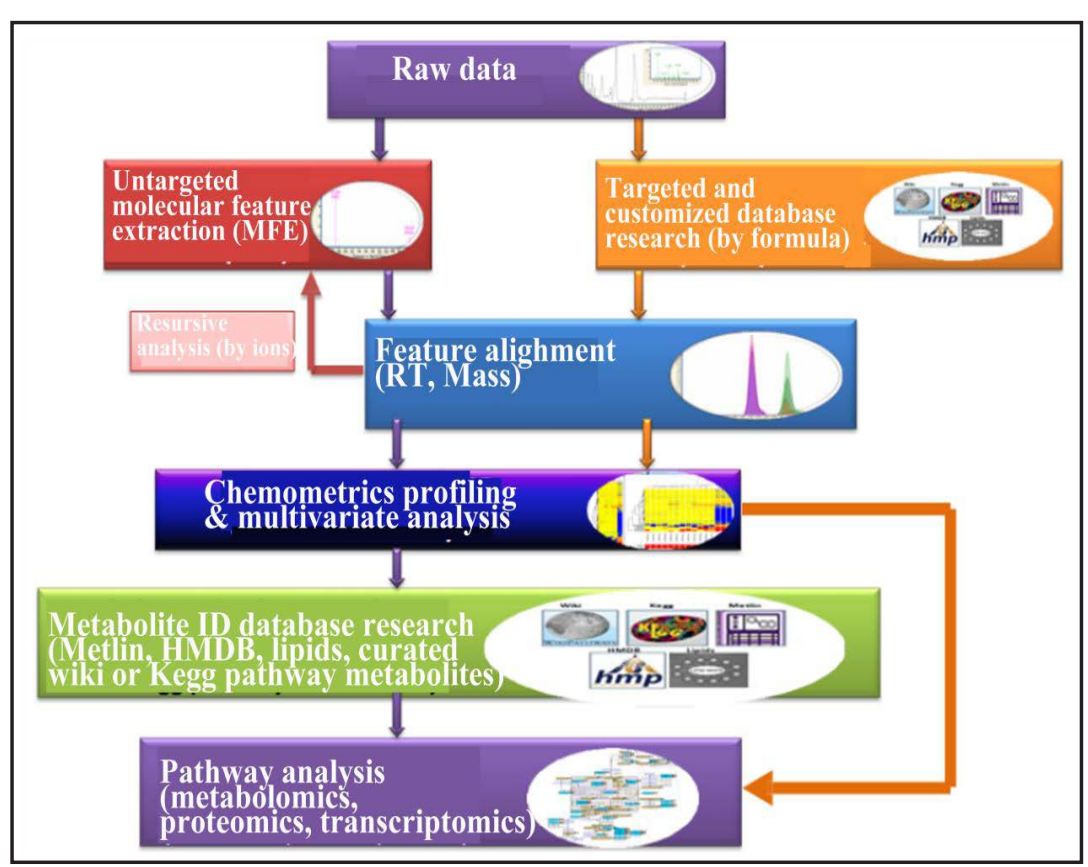

Fig. 1. Screening process of peripheral blood metabolome biomarkers for AD by HPLC-MS. 


\section{Cellular Physiology Cell Physiol Biochem 2017;44:2147-2157 and Biochemistry Published $\begin{aligned} & \text { DOI: 10.1159/000485954 } \\ & \text { C } 2017 \text { The Author(s). Published by S. Karger AG, Basel } \\ & \text { www.karger.com/cpb }\end{aligned}$ \\ Ren et al.: Serum Biomarkers for Aortic Dissection}

fragment analysis. The final results were organized into a two-dimensional data matrix in EXCEL 2007, including variables (rt- $m z$, i.e., the retention time-mass-to-charge ratio), observed quantity (sample), and peak intensity (normalization processing), and the data were entered into SIMCA-P (13.0) for multivariate statistical analysis, as shown in Fig. 1. The mass spectrometry results were analyzed using MPP software to identify metabolites, and metabolites with significant differences were analyzed using Kyoto Encyclopedia of Genes and Genomes (KEGG) for signaling pathway analysis.

\section{Results}

\section{General data}

General data obtained for the three groups are summarized in Table 4. No significant differences were found with regard to gender ratio or mean age.

Instrument calibration and differential analysis of samples in the same group

The Agilent QTOF-6545 instrument was calibrated prior to testing, and stability was examined on the testing day. The results are shown in Fig. 2. The HPLC chromatograms of all six injections overlapped, without any separation, indicating that the instrument was functioning properly with excellent stability. A total of 1426 metabolites were assessed in the 6 tests, and good relative standard deviation (RSD) values were obtained for 1302 .

Ten samples were then randomly selected from each of the three groups, and cluster analysis was performed on the data. As shown in Fig. 3, the consistency of the samples was significantly higher in the AD group than in the other groups, though the correlation of the AD group with the other groups was low. This finding shows that the grouping was very good, and the low correlation between groups indicated that searching for biomarkers based on this test was feasible.

\section{Statistical analysis of samples and distributions of different metabolites}

The data arrays were normalized for more intuitive and reliable results before they were formally analyzed using SMICA-P13.0 software. The principle component analysis (PCA) modeling approach was applied to evaluate differences between two groups, and the results are shown in Fig. 4.

PCA analysis of the three groups showed that the groupings were very good, the associations within the groups were very high, and the differences between groups were large. A strong correlation was found between the NC and HP groups, and a large difference was found between the main components in the AD group and the NC and HP groups. PC1 separated the NC and HP groups from the AD group, indicating that the metabolomes of the NC and HP patients were significantly different from those of the AD patients. PC2 separated the HP group from the NC group, indicating that the metabolomes of the former patients were different from those of the latter. The difference between the NC and AD groups or between the HP and AD groups was larger than the difference between the NC and HP groups.

As presented in Fig. 4B, the corresponding differentially expressed metabolites among the components of the three groups were obtained by PCA characteristic analysis, and the related metabolites were analyzed by database comparison. To validate the differentially expressed metabolites in the three groups, components were analyzed using a volcano plot (Fig. 4C).

As shown in Table 5, 236 differentially expressed metabolites were found in the AD patients compared to the healthy subjects by HPLC-MS analysis, of which 142 were significantly up-regulated and 64 significantly down-regulated. A total of 165 different metabolites were found in the AD patients compared with the patients with hypertension, of which 109 were significantly down-regulated and 56 significantly up-regulated. There were 26 differ- 


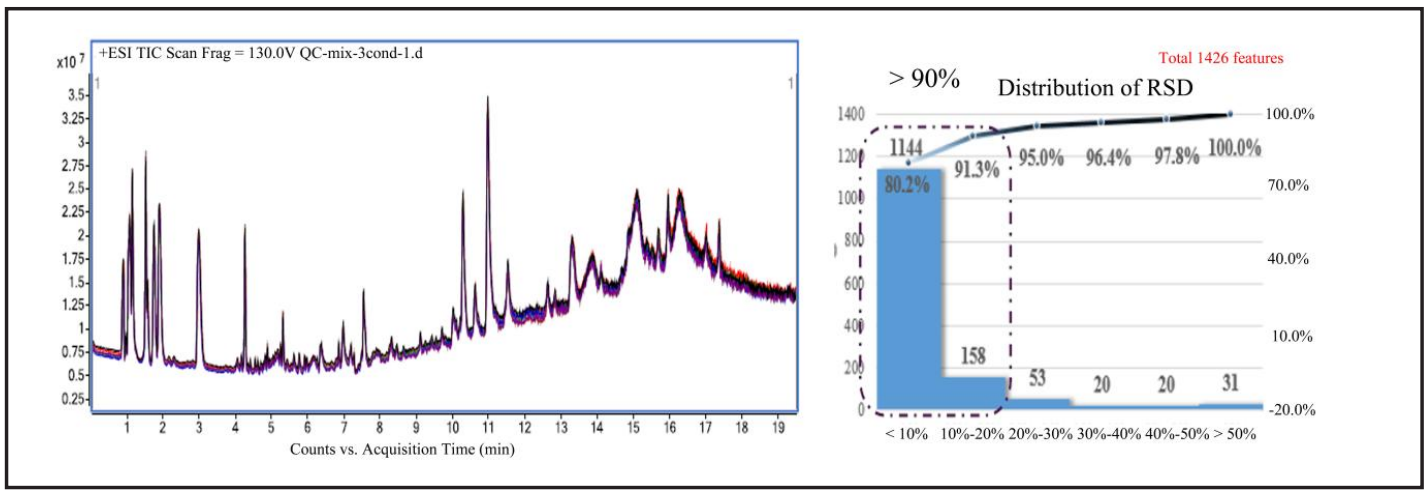

Fig. 2. Results of the instrument stability test. A, Overlapping chromatograms of the sic injections of the mixture of samples from the three groups. B, Test of RSD value distribution.

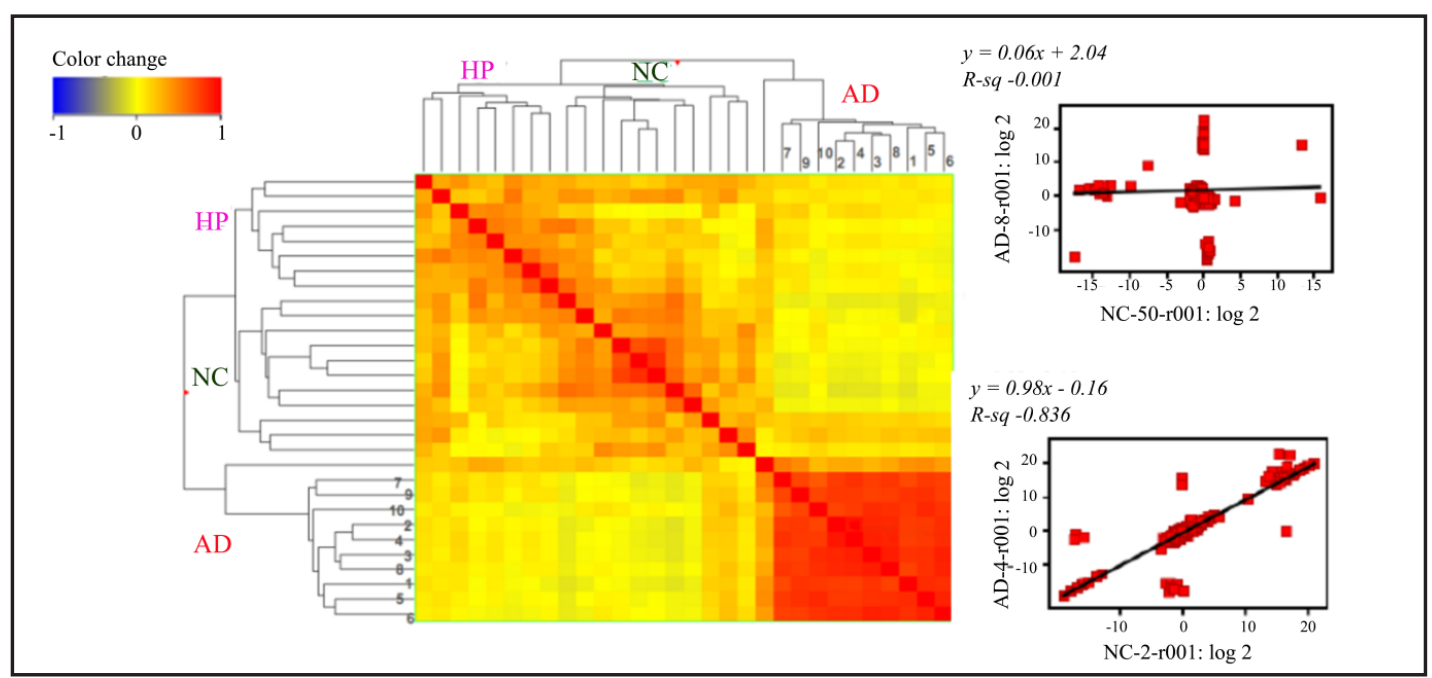

Fig. 3. Differences in metabolites from samples of the same group. Red represents a negative correlation between the two groups of samples; blue represents a positive correlation.

entially expressed metabolites in the hypertensive patients compared with the healthy subjects, of which four were down- and 21 up-regulated. Compared with the NC group, both the $\mathrm{AD}$ and HP groups exhibited differential expression of N,N-dimethyl-safingol, aminopentol, sphinganine, phytosphingosine, bacteriochlorophyllide a, proline betaine, nipecotic acid, phosphinothricin, and Gly Ala Lys (all $P<0.05$ ). Eight metabolites in the peripheral blood of the $\mathrm{AD}$ group were strongly different compared to the $\mathrm{NC}$ and hypertension groups (Table 6); these metabolites were also highly expressed in the peripheral blood of the AD group in comparison with the other components. Among them, three [N1-acetyl-N2-formyl-5-methoxykynuramine (AFMK), glycerophosphocholine, and 2-mercapto histidine betaine (ergothioneine)] showed maximum differences, with peripheral blood levels more than 50 times higher in the AD patients compared with normal human subjects. To further validate the obtained outcomes, the presence of the three substances in each patient of the AD and HP groups relative to the NC group was compared, and the results are summarized in Table 7. The levels of AFMK in the AD group were all significantly higher than those in the HP group, and more than $85 \%$ of the AD patients had significantly higher glycerophosphocholine levels compared to the HP group. Approximately $60 \%$ of the AD patients had significantly higher levels of ergothioneine compared to the HP group, and the remaining $40 \%$ of AD patients had higher or at least not lower levels than the latter. The chemical formulas for AFMK, glycerophosphocholine, and ergothioneine are shown in Fig. 5. These results suggest that the combined use of the three biomarkers has high accuracy for AD screening and diagnosis 


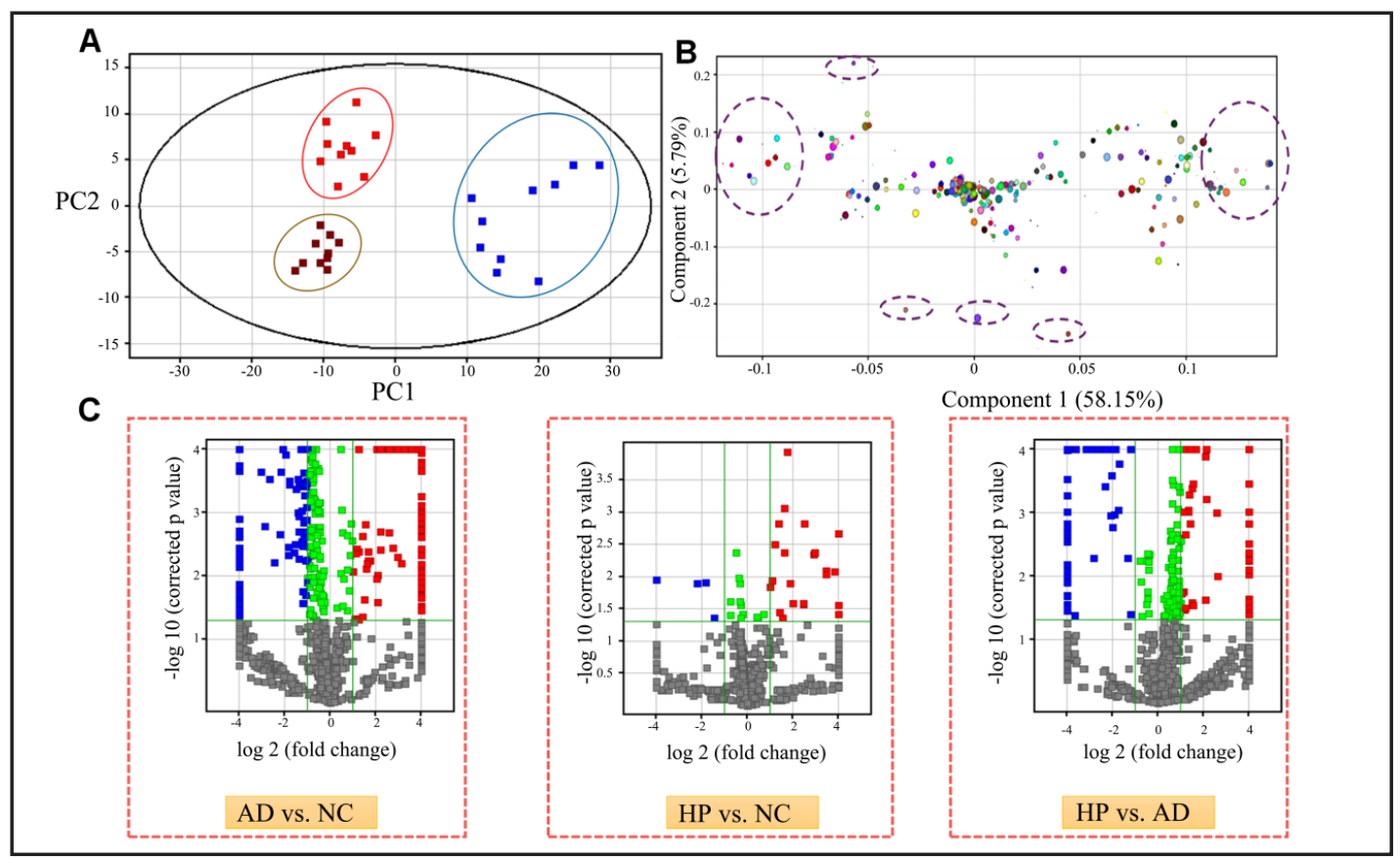

Fig. 4. PCA analysis results showing the components $(A)$ and PCA characteristic analysis of the important compounds for the three groups (B). Distribution of the characteristic metabolites found in PCA characteristic analysis for the three groups in the volcano plot (C), p-value: <0.05; fold change: $>2.0(\log 2)$; in AD vs. NC: red denotes higher in AD and blue lower in AD; in HP vs. NC: red denotes higher in HP and blue lower in HP; in HP vs. AD: red denotes higher in HP and blue lower in HP. NC indicates normal samples, HP indicates hypertension samples, and AD indicates AD samples.

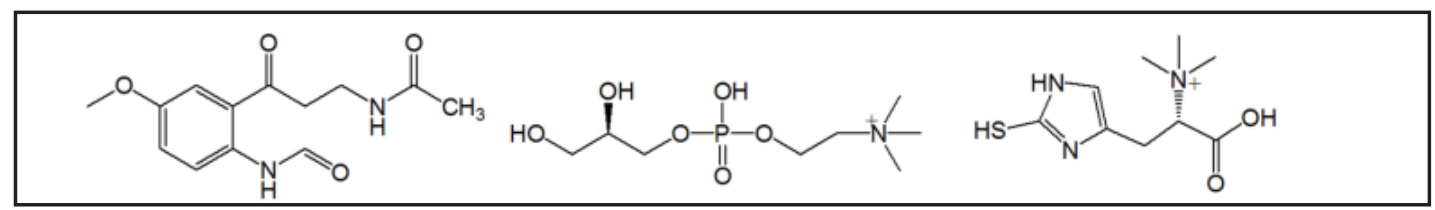

Fig. 5. Characteristic small-molecule biomarkers in the peripheral blood of AD patients: AFMK, glycerophosphocholine, and ergothioneine.

from peripheral blood. Additionally, more than 50 novel substances were identified in the peripheral blood of the AD group, of which two (tetranor-PGE1 and 13, 14-dihydro-19 (R)-hydroxyPGE1) may serve as potential metabolite biomarkers for AD in peripheral blood. These findings will help reveal the pathogenesis of AD.

\section{Potential signaling pathways}

Analysis of the metabolic pathways of the three metabolites with maximally differential expression (AFMK, glycerophosphocholine, and ergothioneine) showed that they are mainly involved in the tryptophan, histidine, glycerophospholipid, ether lipid, and choline metabolic pathways (http://www.kegg.jp/ kegg-bin/search_pathway_text?map=map\&keyword=C00670\&mode=1\&viewImage =true; http://www.kegg.jp/kegg-bin/search_pathway_text?map=map\&keyword=C05642\&mode= 1\&viewImage=true; http://www.kegg.jp/kegg-bin/search_pathway_text?map=map\&keyw ord $=$ C05570\&mode=1\&viewImage=true).
Table 5. Summary of the numbers of differentially expressed metabolites in the peripheral blood of the NC, HP, and AD groups detected by HPLC-MS

\begin{tabular}{lccc}
\hline Comparison & \multicolumn{3}{c}{ Entities } \\
& Down-regulated & Up-regulated & Sum \\
\hline AD vs. NC & 94 & 142 & 236 \\
HP vs. NC & 4 & 21 & 26 \\
HP vs. AD & 109 & 56 & 165 \\
\hline
\end{tabular}




\section{Cellular Physiology Cell Physiol Biochem 2017;44:2147-2157

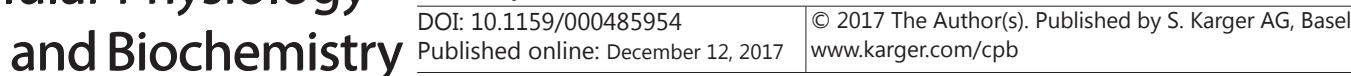 \\ Ren et al.: Serum Biomarkers for Aortic Dissection}

\section{Discussion}

$\mathrm{AD}$ is a serious disease that is generally complicated by hypertension. However, the pathogenesis of $\mathrm{AD}$ is unclear at present, and the changes in whole-body metabolism that are associated with the disease have rarely been reported. AD has an acute onset and is a serious condition; the early mortality of untreated AD is approximately $1 \%-2 \%$ per hour [5]. Moreover, the rate of missed diagnosis for $\mathrm{AD}$ in the emergency department is as high as $50 \%$, and only $25 \%$ of patients with $\mathrm{AD}$ are clearly diagnosed by autopsy after death [5, 9]. Indeed, this delayed diagnosis has become an important factor leading to increased risk of AD-associated morbidity and mortality [7]. For patients with clinical chest and back pain who are suspected of having $\mathrm{AD}$, there is an urgent need to establish a set of highly sensitive and specific clinical indicators for the early diagnosis of $\mathrm{AD}$ in the clinic.

With the recent rapid development of molecular biological technology and the universal application of various new instruments, screening of disease mark-
Table 6. HPLC-MS-detected differentially expressed metabolites in the $\mathrm{AD}$ group, the NC group and the hypertension group

\begin{tabular}{llllll}
\hline Compound & $\begin{array}{l}\text { Peak time } \\
\text { (minutes) }\end{array}$ & $\begin{array}{l}\text { Structural } \\
\text { formula }\end{array}$ & $\begin{array}{l}\text { Matching } \\
\text { score }\end{array}$ & $\begin{array}{l}\text { Molecular } \\
\text { weight }\end{array}$ & CAS number \\
\hline AFMK & 4.943 & C13 H16 N2 04 & 99.31 & 264.1111 & $52450-38-1$ \\
Ergothioneine & 1.103 & C9 H16 N3 02S & 95.36 & 229.089 & $58511-63-0$ \\
Glycerophosphocholine & 1.026 & C8 H21 N 06 P & 98.99 & 257.1028 & $28319-77-9$ \\
TXB1 & 14.864 & C20 H36 06 & 98.56 & 372.2517 & $64626-32-0$ \\
L-Thre0-Sphingosine C-18 & 8.672 & C18 H37 N 02 & 98.43 & 299.2825 & $25695-95-8$ \\
Tetranor-PGE1 & 9.687 & C16 H26 05 & 99.14 & 298.178 & $/$ \\
13,14-Dihydro-19(R)-hydroxyPGE1 & 15.339 & C20 H36 06 & 88.44 & 372.2525 & $/$ \\
Sphingofungin B & 14.866 & C20 H39 N 06 & 84.73 & 389.2774 & $/$ \\
\hline
\end{tabular}

Table 7. The levels of the serum biomarkers in the AD and HP groups (relative to the NC group (fold))

\begin{tabular}{|c|c|c|c|c|c|c|}
\hline \multirow{2}{*}{$\begin{array}{l}\text { Patient } \\
\text { no. }\end{array}$} & \multicolumn{2}{|c|}{ AFMK } & \multicolumn{2}{|c|}{ Glycerophosphocholine } & \multicolumn{2}{|c|}{ Ergothioneine } \\
\hline & HP & $\mathrm{AD}$ & HP & $\mathrm{AD}$ & $\mathrm{HP}$ & $\mathrm{AD}$ \\
\hline 1 & 15 & 150 & 44 & 223 & 22 & 27 \\
\hline 2 & 22 & 173 & 53 & 189 & 17 & 35 \\
\hline 3 & 3 & 167 & 67 & 210 & 28 & 19 \\
\hline 4 & 2 & 200 & 55 & 201 & 20 & 38 \\
\hline 5 & 38 & 201 & 58 & 89 & 33 & 17 \\
\hline 6 & 31 & 401 & 60 & 279 & 18 & 98 \\
\hline 7 & 59 & 98 & No detection & 333 & 40 & 82 \\
\hline 8 & 12 & 339 & 71 & 398 & 19 & 33 \\
\hline 9 & 0.9 & 201 & 20 & 168 & 22 & 109 \\
\hline 10 & No detection & 221 & 21 & 281 & 39 & 90 \\
\hline 11 & 30 & 187 & 49 & 199 & 11 & 16 \\
\hline 12 & 11 & 160 & 71 & 72 & 31 & 189 \\
\hline 13 & 9 & 429 & 79 & 331 & 18 & 176 \\
\hline 14 & 3 & 94 & 42 & 381 & 22 & 153 \\
\hline 15 & 20 & 113 & No detection & 210 & 27 & 132 \\
\hline 16 & 38 & 82 & 66 & 440 & 26 & 40 \\
\hline 17 & 35 & 90 & 38 & 342 & 16 & 104 \\
\hline 18 & 4 & 118 & 77 & 264 & 9 & 15 \\
\hline 19 & 17 & 99 & 62 & 282 & 12 & 113 \\
\hline 20 & 10 & 93 & 66 & 77 & 19 & 99 \\
\hline 21 & No detection & 85 & 61 & 89 & 33 & 148 \\
\hline 22 & 39 & 389 & 30 & 381 & 10 & 112 \\
\hline 23 & 22 & 88 & 41 & 288 & 4 & 88 \\
\hline 24 & 13 & 97 & 44 & 482 & 7 & 69 \\
\hline 25 & 44 & 133 & 43 & 231 & 24 & 93 \\
\hline 26 & 31 & 203 & 62 & 292 & 21 & 118 \\
\hline 27 & 60 & 210 & 66 & 365 & 30 & 69 \\
\hline 28 & 16 & 115 & No detection & 382 & 27 & 26 \\
\hline 29 & 26 & 332 & 80 & 401 & 16 & 17 \\
\hline 30 & 0.8 & 279 & 71 & 392 & 4 & 29 \\
\hline
\end{tabular}

ers, such as protein macromolecules and small-molecule metabolites, is highly desirable. There has been significant progress made in the application of all types of biomarkers for the diagnosis and treatment of diseases, which is useful to clinicians, and molecules such as SMMHC, CK-BB, and MMPs $[14,15]$ have been used in some clinical testing applications. However, there are issues with the application of these biomarkers, inhibiting their widespread implementation for clinical detection. Although smMHC has good sensitivity for early detection of AD, the effective time window is short. Similarly, sFLAF cannot be detected in AD patients with thrombosis within false lumens, even though this molecule is continuously elevated for $72 \mathrm{~h}$. A growing number of studies have found that combined detection of a variety of candidate biomarkers may greatly contribute to the diagnosis of $\mathrm{AD}$, and many peripheral serum markers for $\mathrm{AD}$ patients with prognostic significance, such as D-dimer and serum endothelin [16], have undergone in-depth research. 
In previous studies, the use of peripheral blood biomarkers to diagnose AD showed strong clinical feasibility. For example, these biomarkers can be used for initial screening for $\mathrm{AD}$, and when related indices are abnormal, imaging can be performed for AD confirmation. Such biomarkers can also be used for treatment efficiency follow-up. Additionally, these biomarkers can be used to initially predict patient prognosis. However, previous studies have focused on peripheral blood markers, such as growth factors, enzymes, inflammatory responses, proteins, peptide chain precursors, and other macromolecular proteins [12-15]. Although such studies offer effective information for the early diagnosis and prognosis of $\mathrm{AD}$ and also provide constructive suggestions for $\mathrm{AD}$ pathogenesis elucidation, due to the intrinsic particularity of protein macromolecules, their dissemination in clinical detection for AD faces many challenges, including determining the preservation and testing conditions, diagnosis interval, and cost. Peripheral blood metabolic biomarkers have gained more attention with the development of HPLC-MS technology, our increasing understanding of the metabolic pathways of small molecules, and the gradual perfection of small molecule databases [20-22]. In the present study, metabolites in the peripheral sera of patients with AD were examined by HPLC-MS and compared with corresponding metabolite levels in healthy subjects and hypertensive patients. Eight metabolites were significantly up-regulated in the peripheral sera of AD patients, among which three may be used as potential peripheral blood biomarkers for $\mathrm{AD}$.

A total of 236 differentially expressed metabolites were found in AD patients compared with healthy subjects, of which 142 were significantly up-regulated and 64 significantly down-regulated. In addition, 165 differentially expressed metabolites were found in AD patients compared with patients with hypertension, of which 109 were significantly downand 56 up-regulated. Compared with healthy subjects, 26 differentially expressed metabolites were found in hypertensive patients, with four being down-regulated and 21 up-regulated. Eight metabolites in the peripheral sera of the AD group were differentially expressed compared to the $\mathrm{NC}$ and hypertension groups; these metabolites were also highly expressed in the peripheral sera of the AD group compared with other components. Among them, three metabolites (AFMK, glycerophosphocholine, and ergothioneine) exhibited maximum differences, with levels in AD patient peripheral sera that were more than 50 times higher than in normal human peripheral sera. The combination of these differentially expressed biomarkers may be used for the identification and diagnosis of AD as well as differential diagnosis between $\mathrm{AD}$ and hypertension. Furthermore, more than 50 novel substances were identified in the peripheral sera of the AD group, two of which (tetranor-PGE1 and 13, 14-dihydro-19 (R)-hydroxyPGE1) may serve as potential serum metabolite biomarkers for AD. This finding will contribute to further exploration of AD pathogenesis. Additionally, an increased level of one natural product (sphingofungin B) was found in the AD patient samples compared to the other components, and the presence of a natural product in patient sera is an interesting finding. According to our analysis of the metabolic pathways of the three metabolites with maximally differential expression (AFMK, glycerophosphocholine, and ergothioneine), these differentially expressed metabolites are mainly involved in metabolic pathways underlying tryptophan, histidine, glycerophospholipid, ether lipid, and choline metabolism [23].

This study has some limitations. First, in accordance with the requirement that a biomarker for $\mathrm{AD}$ should be easy to manipulate and have rapid detection, we set the cut-off values at 50 -fold when identifying differently expressed metabolites between the AD and HP groups. However, it remains to be explored whether other differently expressed metabolites can be used as effective biomarkers for AD. Second, due to our limited research capacities and research funding, we did not analyze and compare associations between well-reported protein markers for $\mathrm{AD}$ (such as smMHC, CK-BB, D-dimer, MMPs, and elastin) and the metabolite markers found in this study. Determining whether these metabolite markers are the downstream products of protein markers or whether their accumulation may influence protein expression is the focus of our future research. Additionally, although we found differences between metabolites and pathways in peripheral sera of AD patients, NCs, and 


\section{Cellular Physiology Cell Physiol Biochem 2017;44:2147-2157 \begin{tabular}{l|l|l} 
and Biochemistry Published onlIne: December 12, 2017 & $\begin{array}{l}\text { (c) } 2017 \text { The Author(s). Published by S. Karger AG, Basel } \\
\text { www.karger.com/cpb }\end{array}$
\end{tabular}

hypertensive patients, we did not determine the specific cellular sources of these metabolic substances or differences in their corresponding enzymes and upstream gene levels [24]. An important goal of our next study is to specify the association and differences between these peripheral blood markers and existing protein markers for AD.

\section{Conclusion}

In summary, application of an established detection method to assess changes in peripheral blood AFMK, glycerophosphocholine, and ergothioneine levels in AD patients by HPLC-MS for early diagnosis of AD has good specificity and sensitivity. This method will contribute to the screening and early diagnosis of $\mathrm{AD}$ and can be used to complement the current diagnosis of aortic diseases.

\section{Disclosure Statement}

The authors declare no conflicts of interest.

\section{References}

1 Wang W, Duan W, Xue Y, Wang L, Liu J, Yu S, Yi D: Clinical features of acute aortic dissection from the Registry of Aortic Dissection in China. J Thorac Cardiovasc Surg 2014;148:2995-3000.

-2 Waterford SD, Di Eusanio M, Ehrlich MP, Reece TB, Desai ND, Sundt TM, Myrmel T, Gleason TG, Forteza A, de Vincentiis C, DiScipio AW, Montgomery DG, Eagle KA, Isselbacher EM, Muehle A, Shah A, Chou D, Nienaber CA, Khoynezhad A: Postoperative myocardial infarction in acute type A aortic dissection: A report from the International Registry of Acute Aortic Dissection. J Thorac Cardiovasc Surg 2017;153:521-527.

-3 Regeer MV, Martina B, Versteegh MI, de Weger A, Klautz RJ, Schalij MJ, Bax JJ, Marsan NA, Delgado V: Prognostic implications of descending thoracic aorta dilation after surgery for aortic dissection. J Cardiovasc Comput Tomogr 2017;11:1-7.

4 Chiu P, Miller DC: Evolution of surgical therapy for Stanford acute type A aortic dissection. Ann Cardiothorac Surg 2016;5:275-295.

-5 Ziganshin BA, Dumfarth J, Elefteriades JA: Elefteriades, Natural history of Type B aortic dissection: ten tips. Ann Cardiothorac Surg 2014;3:247-254.

-6 Luebke T, Brunkwall J: Type B Aortic Dissection: A review of prognostic factors and meta-analysis of treatment options. Aorta (Stamford) 2014;2:265-278.

7 Roger VL, Go AS, Lloyd-Jones DM, Adams RJ, Berry JD, Brown TM, Carnethon MR, Dai S, de Simone G, Ford ES, Fox CS, Fullerton HJ, Gillespie C, Greenlund KJ, Hailpern SM, Heit JA, Ho PM, Howard VJ, Kissela BM, Kittner SJ, Lackland DT, Lichtman JH, Lisabeth LD, Makuc DM, Marcus GM, Marelli A, Matchar DB, McDermott MM, Meigs JB, Moy CS, Mozaffarian D, Mussolino ME, Nichol G, Paynter NP, Rosamond WD, Sorlie PD, Stafford RS, Turan TN, Turner MB, Wong ND, Wylie-Rosett J; American Heart Association Statistics Committee and Stroke Statistics Subcommittee: Heart disease and stroke statistics - 2011 update: A report from the American Heart Association. Circulation2011;123:e18-e209.

8 Li Y, Yang N, Duan W, Liu S, Yu S, Yi D: Acute Aortic Dissection in China. Am J Cardiol 2012;110:1056-1061.

-9 De León Ayala IA, Chen YF: Acute aortic dissection: An update. Kaohsiung J Med Sci 2012;28:299-305.

-10 García A, Ferreirós J, Santamaría M, Bustos A, Abades JL, Santamaría N: MR angiographic evaluation of complications in surgically treated type A aortic dissection. Radiographics 2006;26:981-992.

-11 Wang L, Liu S, Yang W, Yu H, Zhang L, Ma P, Wu P, Li X, Cho K, Xue S, Jiang B: Plasma Amino Acid Profile in Patients with Aortic Dissection. Sci Rep 2017;7:40146.

-12 Arrebola JP, Fernández-Rodríguez M, Artacho-Cordón F, Garde C, Perez-Carrascosa F, Linares I, Tovar I, González-Alzaga B, Expósito J, Torne P, Fernández MF, Olea N: Associations of persistent organic pollutants in serum and adipose tissue with breast cancer prognostic markers. Sci Total Environ 2016;566-567:41-49. 


\section{Cellular Physiology Cell Physiol Biochem 2017;44:2147-2157 \begin{tabular}{l|l} 
DOI: 10.1159/000485954 & $\begin{array}{l}\text { O 2017 The Author(s). Published by S. Karger AG, Basel } \\
\text { www.karger.com/cpb }\end{array}$ \\
\hline
\end{tabular} \\ Ren et al.: Serum Biomarkers for Aortic Dissection}

13 Qu F, Zheng SJ, Liu S, Wu CS, Duan ZP, Zhang JL: Serum sphingolipids reflect the severity of chronic HBV infection and predict the mortalityof HBV-acute-on-chronic liver failure. PLoS One 2014;9:e104988.

14 Suzuki T, Katoh H, Watanabe M, Kurabayashi M, Hiramori K, Hori S, Nobuyoshi M, Tanaka H, Kodama K, Sato H, Suzuki S, Tsuchio Y, Yazaki Y, Nagai R: Novel biochemical diagnostic method for aortic dissection. Results of a prospective study using an immunoassay of smooth muscle myosin heavy chain. Circulation 1996;93:1244-1249.

15 Taniyama M, Yoh S, Asaba Y, Maruyama T, Takei I, Kataoka K: Elevated serum creatine kinase level in diabetic patients with nephrotic syndrome: A role of fluid retention. Ann Intern Med 1987;106:711.

-16 Apostolakis E, Akinosoglou K: What's new in the biochemical diagnosis of acute aortic dissection: Problems and perspectives. Med Sci Monit 2007;13:RA154-158.

17 Li H, Li J, Wang Y, Yang T: Proteomic analysis of effluents from perfused human heart for transplantation: identification of potential biomarkers for ischemic heart damage. Proteome Sci 2012;10:21.

18 Liu Y, Qing H, Deng Y: Biomarkers in Alzheimer's disease analysis by mass spectrometry-based proteomics. Int J Mol Sci 2014;15:7865-7882.

19 Tessitore A, Gaggiano A, Cicciarelli G, Verzella D, Capece D, Fischietti M, Zazzeroni F, Alesse E: Serum Biomarkers Identification by Mass Spectrometry in High-Mortality Tumors. Int J Proteomics 2013;2013:125858.

20 Sarah SA, Faradalila WN, Salwani MS, Amin I, Karsani SA, Sazili AQ: LC-QTOF-MS identification of porcinespecific peptide in heat treated pork identifies candidate markers for meat species determination. Food Chem 2016;199:157-164.

21 Wen JJ, Zago MP, Nuñez S, Gupta S, Burgos FN, Garg NJ: Serum proteomic signature of human Chagasic patients for the identification of novel potential protein biomarkers of disease. Mol Cell Proteomics 2012;11:435-452.

-22 White L, Ma J, Liang S, Sanchez-Espiridion B, Liang D: LC-MS/MS determination of d-mannose in human serum as a potential cancer biomarker. J Pharm Biomed Anal 2017;137:54-59.

-23 Liu W, Wang B, Wang T, Liu X, He X, Liu Y, Li Z, Zeng H: Ursodeoxycholic Acid Attenuates Acute Aortic Dissection Formation in Angiotensin II-Infused Apolipoprotein E-Deficient Mice Associated with Reduced ROS and Increased Nrf2 Levels. Cell Physiol Biochem 2016;38:1391-1405.

-24 Marchon C, de Marco Ornelas E, da Silva Viegas KA, Lacchini S, de Souza RR, Fonseca FL, Maifrino LB: Effects of moderate exercise on the biochemical, physiological, morphological and functional parameters of the aorta in the presence of estrogen deprivation and dyslipidemia: an experimental model. Cell Physiol Biochem 2015;35:397-405. 